\title{
Bilateral Facial Paralysis and Otitis Media as the First Presentations of Wegener's Granulomatosis: A Case Report
}

\author{
Seyed Mohammad Javad Hosseini ${ }^{1}$, Jaleh Yousefi ${ }^{2}$, Habib Yaribeygi ${ }^{3}$, Gholam Hossein Alishiri ${ }^{4 *}$ \\ ${ }^{1}$ Biological-Molecular Research Center, Baqiyatallah University of Medical Sciences, Tehran, Iran \\ ${ }^{2}$ Department of Otorhinolaryngology, Faculty of Medicine, Baqiyatallah University of Medical Sciences, Tehran, Iran \\ ${ }^{3}$ Health Research Center, Baqiyatallah University of Medical Sciences, Tehran, Iran \\ ${ }^{4}$ Department of Rheumatology, Faculty of Medicine, Baqiyatallah University of Medical Sciences, Tehran, Iran
}

*Corresponding Author: Gholam Hossein Alishiri, M.D., Professor, Department of Rheumatology, Faculty of Medicine, Baqiyatallah University of Medical Sciences, Tehran, Iran. Tel: +98-21-81263618, Email: ghalishiri@gmail.com

Received September 4, 2017; Accepted September 26, 2017; Online Published October 14, 2017

\begin{abstract}
Introduction: Cranial nerve palsy in Wegener's granulomatosis is a curious incident, particularly if it occurs without kidney or lung involvement. In a review of medical articles, only 1 case of Wegener's granulomatosis with bilateral facial nerve palsy was found.

Case Presentation: The patient was a 16-year-old female who presented with pain and hearing loss in both ears and reduced muscle tone in the right side of her face. After myringotomy and ventilation tube embedding in both ears, she gradually developed infectious otorrhea in both ears. Despite antibiotic and antifungal therapy for 2 weeks after surgery, the patient developed paresis in the left facial nerve and gag reflex disorder. Both magnetic resonance imaging (MRI) and magnetic resonance venography (MRV) tests were normal, but the C-anti-neutrophil cytoplasmic antibody (CANCA) test result was about forty times higher than normal, and a sinus biopsy also verified Wegener's granulomatosis. The patient was then treated with pulse corticosteroid and cyclophosphamide therapy. Her gag reflex healed and the ear secretions stopped, but the facial nerve palsy continued.

Conclusion: Although cranial nerve involvement in Wegener's granulomatosis is an unusual incidence, it may occur in some cases. In the current case, otitis media and cranial nerve paresis without kidney or lung disorders were the only signs of disease onset; of course, computerized tomography (CT) scan revealed a right mandibular sinus disorder without any clinical signs. Another important point in this case is that other identified Wegener's patients were in their fourth or fifth decade of life; thus, Wegener's was the last choice for a diagnosis in the current case.

Keywords: Otitis, Granulomatosis, Wegener Syndrome, Paresis, Facial Nerve
\end{abstract}

\section{Introduction}

Wegener's granulomatosis is a necrotic granuloma disorder with unknown etiology and vasculitis accompanied by inflammation and different tissue damage as well..$^{1}$ This disease is, in fact, a systemic vasculitis of both small and medium arteries as well as venules and sometimes large arteries. $^{2}$ In the classic form of the disease, the upper and lower airways, respiratory tract, and kidneys are primarily involved. However, it can also engage other organs, such as the eyes, ears, skin, jaws, and joints, and it spreads throughout the days and months. ${ }^{3}$ In $90 \%$ of cases, the disease begins with respiratory involvement and is often controlled by implementing proprietary treatment protocols. Delayed treatment and control of the inflammatory processes endangers life. ${ }^{4}$ Choosing the type of treatment depends on the affected organ and the severity of the disease. ${ }^{5}$ Treatment is usually initiated with glucocorticoids such as prednisolone and cytotoxic drugs such as cyclophosphamide, methotrexate, or azathioprine. ${ }^{5}$

This rare syndrome has an incidence rate of about 3 in 100000 with a higher occurrence rate in the fourth and fifth decades of life in both men and women. ${ }^{6}$ In the case presented here, however, the disease occurred in a younger subject and had an unusual progression.

\section{Case Presentation}

2.1. Patient Information

The patient was a 16-year-old female who presented with

Copyright $(2017$ The Author(s). This is an open-access article distributed under the terms of the Creative Commons Attribution License (http:// creativecommons.org/licenses/by/4.0), which permits unrestricted use, distribution, and reproduction in any medium, provided the original work is properly cited. 
pain and hearing loss in both ears and reduced muscle tone in the right side of her face. The hearing loss had started first in her right and then her left ear 2 months before admission. One month before admission, she experienced muscle paresis in the right side of her face and severe pain in her right ear. During this period, she was treated for otitis and sinusitis, but showed no improvement.

\subsection{Clinical Findings}

During the patient's first exam, serious otitis media with $30 \mathrm{db}$ conductive hearing loss and type B tympanometry in both ears. In her nose, mucous secretions could be seen, but her throat was normal. The left facial nerve was normal, but the patient could not close her right eye completely, and she had no movement in either lip. Because of treatment failure, middle ear effusion, and facial nerve paresis, the patient was considered for myringotomy and tube ventilation in both ears. The lab data before surgery was: Hb 10.4 (with low mean corpuscular volume [MCV]), White blood cell (WBC): 16000 (80\% neutrophils) and ESR 20.

\subsection{Therapeutic Interventions}

The patient underwent surgery. One day after surgery, she had severe tremoring in her hands and feet while standing which was relieved by resting in bed and nasal oxygen therapy. The patient also had inspiratory and expiratory stridor. Examination revealed only right side facial paresis; electroencephalography (EEG), MRV, and brain MRI were all normal. The assessment of facial nerve function and electroneuronography (ENOG) test results showed that the patient's right side had 35\% function in comparison to the left side.

Despite receiving ceftriaxone, the patient developed putrid secretions from her left ear on the third day after surgery and from her right ear on the fourth day. On the fifth day, ear pain was added. The culture results from her ears showed candida albicans, and oral fluconazole was started $(100 \mathrm{mg} / 12 \mathrm{~h})$ with topical clotrimazole $2 \%$. Ceftriaxone was discontinued, and ceftazidime, clindamycin, vancomycin, and amphotericin B were started. The ear secretions gradually decreased, but her pain showed no response to treatment.

\subsection{Follow-up and Outcomes}

Audiometry showed $60 \mathrm{db}$ conductive hearing loss in both ears. On the 10th day after surgery, the patient developed bilateral facial paresis and swallowing dysfunction. Physical examination revealed a very weak gag reflex. To rule out mucormycosis, tumors at her skull base, leukemia, Wegener's, sarcoidosis, and cavernous sinus thrombosis, an MRI, MRV, and skull base and nasopharynx CT scans were performed. No abscesses, masses, or infections in the brain were noted, but the maxillary CT scan showed right maxillary sinus haziness. Upon exam, there was nasal bleeding, especially on the right side. Therefore, the necessary tests for Wegener's granulomatosis and sarcoidosis were conducted. The patient's inferior turbinate and nasal mucosa were biopsied for Wegener's and mucormycosis. Lung CT scan and U/A tests were normal. antinuclear antibodies (ANA) and P-anti-neutrophil cytoplasmic antibody (PANCA) tests were normal, but $\mathrm{C}$-anti-neutrophil cytoplasmic antibody (CANCA) measured 40 times higher than normal value. Biopsy identified vasculitis with granuloma, but for mucus the results were negative.

With these findings, Wegener's granulomatosis without lung and kidney involvement was diagnosed. Consequently, pulse steroid and cyclophosphamide therapies were started. In follow-up, the patient had near normal hearing without pain. After 2 months, her gag reflex became normal. In the fifth month of follow-up after treatment and with physiotherapy, the patient's facial paresis showed relative improvement.

\section{Discussion}

Wegener's granulomatosis is a systemic vasculitis; in classic and common forms, it has both kidney and lung involvement. ${ }^{2}$ In rare cases (such as in the current patient), it develops with unusual symptoms such as hearing loss and bilateral involvement of the facial nerve at an unusual age. $^{3}$ Thus, early diagnosis with rapid and wise treatment accompanied by rule out of any important differential.

Early detection and diagnosis plus proper treatment can be life-saving and prevent morbidity and pathological processes. $^{3} \quad$ Wegener's characteristic features are involvement of the granulomatous vasculitis in upper and lower airways with glomerulonephritis. Also, diffusive vasculitis can be seen in various degrees in smaller veins and arteries. The average age for this disease is about 40 years. ${ }^{7}$ However, about $15 \%$ of subjects are younger than 19 years. ${ }^{8}$ Wegener's vasculitis has many different clinical aspects. According to the National Institute of Health (NIH), the most common organs involved at first are the sinuses, lungs, joints (arthralgia and arthritis), ears (otitis media), and kidneys. ${ }^{9}$ The central nervous system and peripheral nerves are uncommon sites of involvement.

In the presented case, the patient's first detected symptoms were otitis media and facial paralysis without other organ involvement. In a literature review, only 1 case occurring at a young age was found, and that patient was a 23-year-old woman with bilateral recurrent otitis media, hearing loss, bilateral facial paralysis, and lung involvement. ${ }^{10}$ In the patient presented herein, nose and middle ear biopsies were normal, but the lung biopsy was positive. ${ }^{10}$ The CANCA test was also positive in the current case.

\section{Conclusion}

Based on this study and because early diagnosis and preventive therapies result in better outcomes, it is concluded that Wegener's granulomatosis should be considered as a possible diagnosis and/or ruled out in younger patients with chronic otitis and facial paralysis. 


\section{Authors' Contributions}

All authors contributed equally to this study.

\section{Conflicts of Interest}

The authors declare that they have no conflicts of interest with this study.

\section{Ethical Approval}

The current study was approved by the Ethics Committee of a local hospital. The patient and her parents gave informed consent for the patient's participation in this study.

\section{Acknowledgments}

The authors express their gratitude to the patient and her parents. Furthermore, they wish to thank the Clinical Research Development Unit of Baqiyatallah Hospital for their technical support.

\section{References}

1. Etanercept plus standard therapy for Wegener's granulomatosis. N Engl J Med. 2005;352(4):351-361. doi:10.1056/ NEJMoa041884.

2. Comarmond C, Cacoub P. Granulomatosis with polyangiitis (Wegener): clinical aspects and treatment. Autoimmun Rev. 2014;13(11):1121-1125. doi:10.1016/j.autrev.2014.08.017.

3. Bohm M, Gonzalez Fernandez MI, Ozen S, et al. Clinical features of childhood granulomatosis with polyangiitis (wegener's granulomatosis). Pediatr Rheumatol Online J. 2014;12:18. doi:10.1186/1546-0096-12-18.

4. Girard C, Charles P, Terrier B, et al. Tracheobronchial stenoses in granulomatosis with polyangiitis (Wegener's): a report on 26 cases. Medicine (Baltimore). 2015;94(32):e1088. doi:10.1097/ md.0000000000001088.

5. Lutalo PM, D'Cruz DP. Diagnosis and classification of granulomatosis with polyangiitis (aka Wegener's granulomatosis). J Autoimmun. 2014;48-49:94-98. doi:10.1016/j.jaut.2014.01.028.

6. Liu X, Cui Y, Li Y, Wang C, Zhao H, Han J. Using inpatient data to estimate the prevalence of Wegener's granulomatosis in China. Intractable Rare Dis Res. 2016;5(1):31-35. doi:10.5582/ irdr.2015.01015.

7. Tan LT, Davagnanam I, Isa $\mathrm{H}$, et al. Clinical and imaging features predictive of orbital granulomatosis with polyangiitis and the risk of systemic involvement. Ophthalmology. 2014;121(6):1304-1309. doi:10.1016/j.ophtha.2013.12.003.

8. Guneyli S, Ceylan N, Bayraktaroglu S, et al. Imaging findings of pulmonary granulomatosis with polyangiitis (Wegener's granulomatosis): lesions invading the pulmonary fissure, pleura or diaphragm mimicking malignancy. Wien Klin Wochenschr. 2016;128(21-22):809-815. doi:10.1007/s00508-015-0747-1.

9. Hoffman GS, Kerr GS, Leavitt RY, et al. Wegener granulomatosis: an analysis of 158 patients. Ann Intern Med. 1992;116(6):488498. doi:10.7326/0003-4819-116-6-488.

10. Nikolaou AC, Vlachtsis KC, Daniilidis MA, Petridis DG, Daniilidis IC. Wegener's granulomatosis presenting with bilateral facial nerve palsy. Eur Arch Otorhinolaryngol. 2001;258(4):198-202. doi:10.1007/s004050100327. 